archives-ouvertes

\title{
Uncertainty Analysis for Day Ahead Power Reserve - Quantification in an Urban Microgrid Including PV Generators
}

\author{
Xingyu Yan, Dhaker Abbes, Bruno François
}

\section{To cite this version:}

Xingyu Yan, Dhaker Abbes, Bruno François. Uncertainty Analysis for Day Ahead Power Reserve Quantification in an Urban Microgrid Including PV Generators. Renewable Energy, Elsevier, 2017. hal-01756824

HAL Id: hal-01756824

https: / hal.archives-ouvertes.fr/hal-01756824

Submitted on 3 Apr 2018

HAL is a multi-disciplinary open access archive for the deposit and dissemination of scientific research documents, whether they are published or not. The documents may come from teaching and research institutions in France or abroad, or from public or private research centers.
L'archive ouverte pluridisciplinaire HAL, est destinée au dépôt et à la diffusion de documents scientifiques de niveau recherche, publiés ou non, émanant des établissements d'enseignement et de recherche français ou étrangers, des laboratoires publics ou privés. 


\title{
Quantification in an Urban Microgrid Including PV Generators
}

\author{
Xingyu YAN ${ }^{1}$, Dhaker ABBES ${ }^{2}$, Bruno FRANCOIS ${ }^{1}$ \\ Univ. Lille, Centrale Lille, Arts et Métiers Paristech, HEI, EA 2697 - L2EP - Laboratoire d’Electrotechnique et d'Electronique de Puissance, \\ F-59000 Lille, France \\ E-mail: bruno.francois@ec-lille.fr
}

\begin{abstract}
Setting an adequate operating power reserve (PR) to compensate unpredictable imbalances between generation and consumption is essential for power system security. Operating power reserve should be carefully sized but also ideally minimized and dispatched to reduce operation costs with a satisfying security level. Although several energy generation and load forecasting tools have been developed, decision-making methods are required to estimate the operating power reserve amount within its dispatch over generators during small time windows and with adaptive capabilities to markets, as new ancillary service markets. This paper proposes an uncertainty analysis method for power reserve quantification in an urban microgrid with a high penetration ratio of PV (photovoltaic) power. First, forecasting errors of PV production and load demand are estimated one day ahead by using artificial neural networks. Then two methods are proposed to calculate one day ahead the net demand error. The first perform a direct forecast of the error, the second one calculates it from the available PV power and load demand forecast errors. This remaining net error is analyzed with dedicated statistical and stochastic procedures. Hence, according to an accepted risk level, a method is proposed to calculate the required PR for each hour.
\end{abstract}

Index Terms - Power reserve scheduling; renewable energy sources; forecast errors; uncertainty analysis; reliability.

\section{I.INTRODUCTION}

NCREASE of the electricity produced by renewable energy sources (RES) contributes to energy supply portfolio diversity and

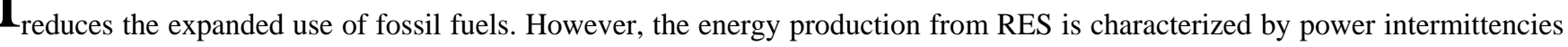
and production uncertainties, especially for PV and wind power. New operational challenges appear for grid operators like ramping and regulation requirements in addition to impacts on power system stability. Hence, grid codes evolve and in many European countries, renewable sources are more and more required to provide ancillary services for grid operators [1]. In order to maintain the security and reliability of grids with a high share of renewable generators, primary, secondary and tertiary regulation as well as spinning reserve are now required from renewable generators in more and more grid codes [2, 3]. This operating power reserve should be ideally minimized to reduce system costs with a satisfying security level.

Typically, PV power generation forecasting is needed to optimize the operation and to reduce the cost of power systems, especially for the scheduling and dispatching of required hourly operating [4]. However, the predicted uncertainty associated with forecast cannot be eliminated even with the best model tools. In addition to the load demand uncertainty, the combination of power generation and consumption variability with forecast uncertainty makes the situation more difficult for power system operators to schedule and to set power reserve level. Therefore, the uncertainties from both generation and consumption must be taken into account by an accurate stochastic model for power system management. In addition, forecasting errors from system uncertainty analysis could be used to set power reserve [5].

Historically, most conventional utilities have adopted deterministic criteria for the reserve requirement: the operating rules required PR to be greater than the capacity of the largest on-line generator or a fraction of the load, or equal to some function of both of them. Those deterministic criteria are wildly used because of their simplicity and understandable employing. However, 
these deterministic calculation methods are gradually replaced by probabilistic methods that respond to the stochastic factors corresponding to the system reliability. Several research works have been focused on calculating the total system uncertainty from all the variable sources. Based on dynamic simulations, the study in [8] is focusing on dynamic frequency control of an isolated system and the reduction of the impact due to large shares of wind and PV powers. However this work did not consider other aspects, such as variability and forecast accuracy. A deterministic approach is proposed in [9] to analyze the flexibility of thermal generation to balance wind power variations and prediction errors. A stochastic analysis could improve it in order to be able to quantify the power reserve with a risk index. A stochastic model was developed in [10] to simulate the operations and the line disconnection events of the transmission network due to overloads beyond the rated capacity. But the issue is clearly that analysis of the system states, in terms of power request and supply, are critical for network vulnerability and may induce a cascade of line disconnections leading to a massive network blackout. An insurance strategy is proposed in [11] to cover the possible imbalance cost that wind power producer may incur in electricity markets. Monte Carlo simulations have been used to estimate insurance premiums for further analysis excesses and so require a significant calculation time.

Our previous works in [6, 7] showed that forecasting errors from system uncertainty analysis could be used for PR setting. Following these promising results and experiences, we have carried out further investigations on rigorous methods to quantify the required PR. The task is to calculate it by considering uncertainties from PV prediction and load forecast or with uncertainty estimation. In the second part of this paper, PV power and load uncertainty and variability are analyzed. Then, the artificial neural network based prediction methods are applied to forecast PV power, load demand and errors. In the third part, the Net Demand (ND) Forecasted uncertainty is obtained, for each hour of the next day, as the difference between the forecasted production uncertainty and the forecasted load uncertainty. Two methods are detailed to calculate the ND forecast errors. An hourly probability density function of all predicted ND forecasted errors has been used for the error analysis. In the fourth part, a method is explained to assess the accuracy of these predictions and to quantify the required operating PR to compensate the system power unbalancing due to these errors. Power reserve is obtained by choosing a risk level related to two reliability assessment indicators: loss of load probability (LOLP), and expected energy not served (EENS). Finally, this management tool is proved through an illustrative example.

\section{II.METHODOLOGY}

\section{A.PV Power and Load Uncertainty Analysis}

The PV power variability is the expected change in generation while PV power uncertainty is the unexpected change from what was anticipated, such as a suddenly cloud cover. The former depends on the latitude and the rotation of Earth, while the latter is mostly caused by uncertainty conditions, such as cloud variations over the PV. The movement of clouds introduces a significant uncertainty that can result in rapid fluctuations in solar irradiance and therefore PV power output. However, the influence of a moving cloud and, hence, the shading of an entire PV site depends on the PV area, cloud speed, cloud height and many other factors. Data from solar installations covering a large spatial extent have an hourly temporal dynamic, while individual zones have instantaneous dynamics as in local distribution networks or micro grids.

The daily operation of a power system should be matched to load variations to maintain system reliability. This reliability refers to two areas:

- $\quad$ system adequacy, which depends on sufficient facilities within the system to satisfy system operational constraints and load demand, and

- $\quad$ system security, which is the system ability to respond to dynamic disturbances. 
When RES represent a significant part of the power generation, system operating power reserve must be larger to regulate the variations and maintain the security level. This additional power is required to stable the electrical network. Classically this power reserve is provided by controllable generators (gas turbines, diesel plant, ..). Today, the increasing of balancing power reserves leads to a significant increase in the power system operating cost and so system may limit the PV power penetration is due to the variability and uncertainty over short time scales.

There are different ways to manage variability and uncertainty. In general, system operators and planners use mechanisms including forecasting, scheduling, economic dispatch, and power reserves to ensure performances that satisfy reliability standards with the least cost. The earlier system operators and planners know what kind of variability and uncertainty they will have to deal with, the more options they will have to accommodate it and cheaper it will be. The key task of variability and uncertainty management is to maintain a reliable operation of the power system (grid connected or isolated) while keeping down costs.

Energy management of electrical systems is usually implemented over different time scales. One day ahead, system operators have to balance the load demand with electrical generation by planning the starting and set points of controllable generators on an hourly time step. Risks are also considered and thus a power reserve has also to be hourly planned. During the day, unexpected PV power lack is compensated by injecting a primary power reserve. The PV variability can be separated into different time scales associated with different impacts, onto the grid management and costs. Consequently, more capacity to compensate errors in forecasts or unexpected events must be accommodated.

The instantaneous PV power output is affected by many correlated external and physical inputs, such as irradiance, humidity, pressure, cloud cover percentage, air/panels temperature and wind speed. The per unit surface power output is modeled by [12]:

$P_{P V}(t)=\eta \cdot A \cdot I_{r}(t) \cdot\left(1-C_{p} \cdot(T(t)-25)\right)$

where $\eta$ is the power conversion efficiency of the module (\%), $A$ is the surface area of PV panels $\left(\mathrm{m}^{2}\right), I_{r}$ is the global solar radiation $\left(\mathrm{kW} / \mathrm{m}^{2}\right)$ and $T$ is the outside air temperature $\left({ }^{\circ} \mathrm{C}\right), C_{p}$ is the cell maximum power temperature coefficient (equal to 0.0035 but it can varies from 0.005 to 0.003 per ${ }^{\circ} \mathrm{C}$ in crystalline silicon).

The PV power, solar irradiance and temperature of our lab PV plant have been recorded during three continuous days (22/06/2010 - 24/06/2010) and are presented respectively in Fig. 1. The PV power variability is highly correlated with irradiance, so as to the temperature, while the PV power uncertainty is almost caused by the irradiance change. Sensed PV power data points can be drawn according to sensed irradiance and temperature data points in order to highlight correlations (Fig. 2).

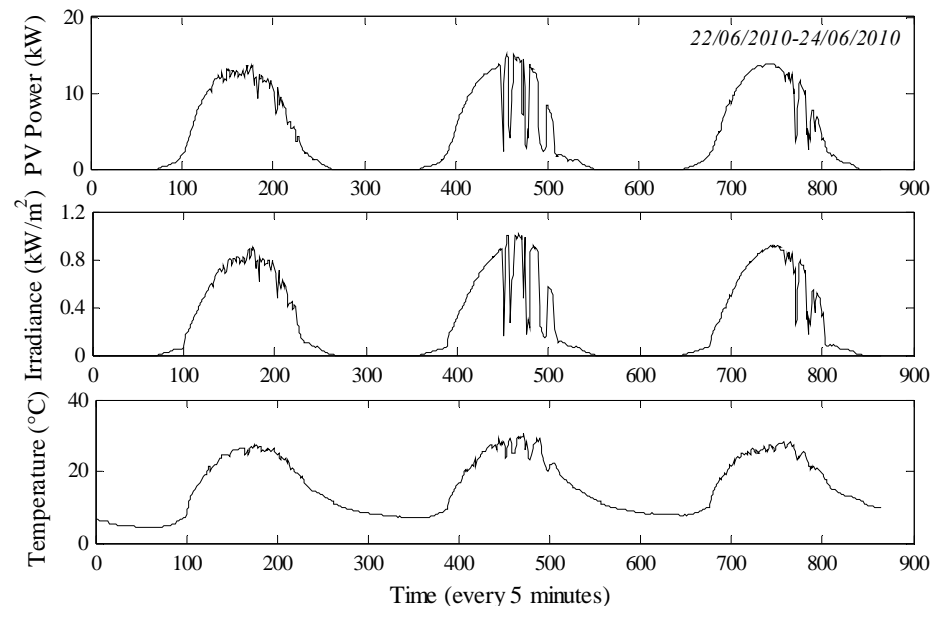




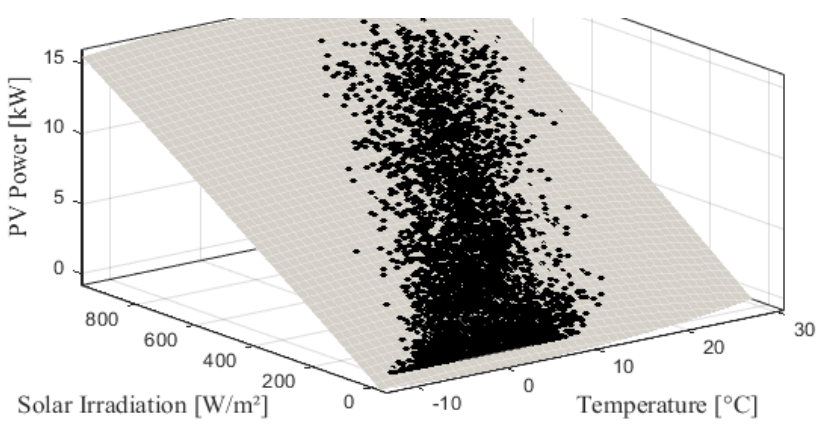

Fig. 2. Irradiance and Temperature vs. Power correlation.

The local load consumption demand is also highly unpredictable and quite random. It depends on different factor, such as the economy, the time, the weather, and other random effects. However, for power system planning and operation, load demand variation and uncertainty analysis are crucial for power flow study or contingency analysis. As for PV production, load demand variations exist in all time scales and system actions are needed for power control in order to maintain the balancing.

\section{B.Power Forecasting Methodology}

\section{1)PV Power Forecasting}

In recent decades, several forecasting models of energy production have been published [13-17]. For PV power, one method consists in forecasting solar radiation and then forecasting PV power with a mathematical model of the PV generator. A second one proposes to directly predict the PV power output from environmental data (irradiance, temperature, etc.). Statistical analysis tools are generally used, such as linear/multiple-linear/non-linear regression and autoregressive models that are based on time series regression analysis [18]. These forecasting models rely on modeling relationships between influent inputs and the produced output power. Consequently, mathematical model calibration and parameters adjustment process take a long time. Meanwhile, some intelligent based electrical power generation forecast methods, as expert systems, fuzzy logic, neural networks, are widely used to deal with uncertainties of RES power generation and load demand [13,19].

In daily markets, the hourly PV power output for the next day (day $\mathrm{D}+1$ ) at time step $h$ is represented as the sum of a day ahead hourly forecast PV power $\left(\widetilde{P} v_{h}\right)$ and the forecast error $\left(\varepsilon_{h}{ }^{P v}\right)$ :

$$
P v_{h}=\tilde{P} v_{h}+\varepsilon_{h}^{P v}
$$

\section{2)Load Demand Forecasting}

For load demand forecast, numerous variables affect directly or indirectly the accuracy. Until now, many methods and models have already been tried out. In [19], several long-term (month or year) load forecasting methods are introduced and are very important for planning and developing future generation, transmission and distribution systems. In [27], a long term probabilistic load forecasting method is proposed with three modernized elements: predictive modeling, scenario analysis, and weather normalization. Long-term and short-term load forecast play important roles in the formulation of secure and reliable operating strategies for the electrical power system. The objective is to improve the forecast accuracy in order to optimize power system planning and to reduce costs.

The day ahead actual load demand at time step $h\left(L_{h}\right)$ is assumed to be the sum of the day ahead forecasted load ( $\widetilde{L}_{h}$ ) and an error $\left(\varepsilon_{h}^{L}\right)$ :

$L_{h}=\tilde{L}_{h}+\varepsilon_{h}^{L}$ 
3)Net Demand Forecasting

Knowing the PV power forecasting and the load demand forecasting, the net demand forecasting ( $\left.\tilde{N} D_{h}\right)$ for a given time step $h$ is expressed as:

$\tilde{N} D_{h}=\tilde{L}_{h}-\tilde{P} v_{h}$

The real net demand $\left(N D_{h}\right)$ is composed of the forecasted day ahead ND and a forecast error $\left(\varepsilon_{h}^{N D}\right)$ :

$N D_{h}=\tilde{N} D_{h}+\varepsilon_{h}^{N D}$

\section{C.Application of Back-Propagation ANN to Forecast}

In order to predict the net demand errors, as well as PV and load forecast errors, we have developed several back-propagation (BP) Artificial Neural Networks (ANN) [22]. Compared with conventional statistical forecasting schemes, ANN has some additional advantages, such as simplicity in adaptability to online measurements, data error tolerance and lack of any excess information. Since the fundamentals of ANN based predictors can be found in many sources, it will not be recalled again.

\section{III.NET DEMAND UNCERTAINTY ANALYSIS}

\section{A.Net Demand Uncertainty}

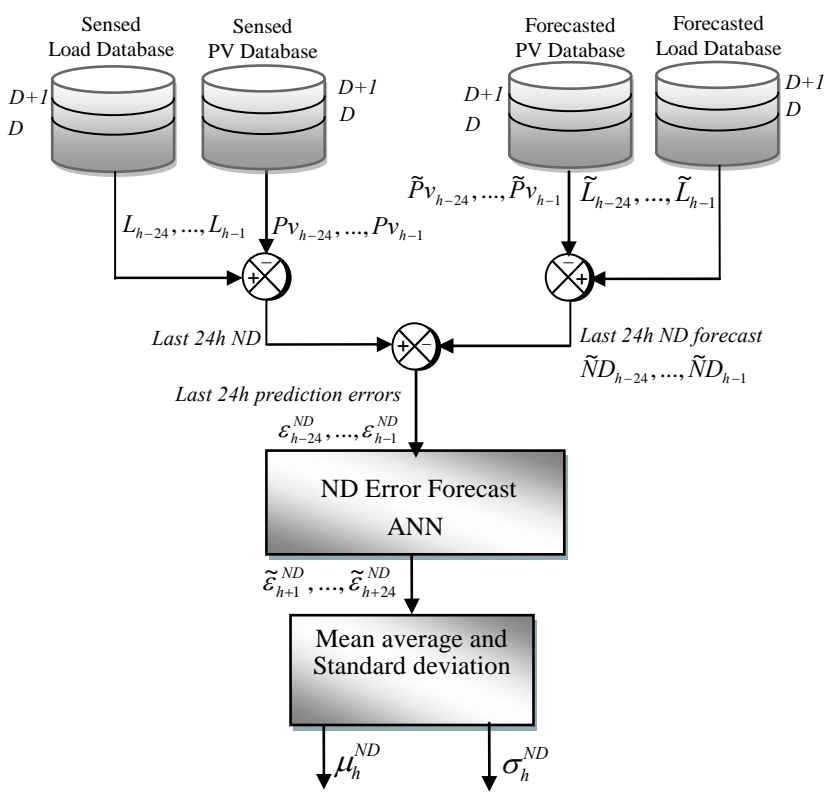

Fig. 3. Net demand uncertainty calculation from ND error forecast.

In order to simplify the study, the uncertainties coming from conventional generators and network outages are ignored and only load and PV power uncertainties are considered. Then the error of the ND forecasting is representing the ND uncertainty. Two possible methods are proposed to calculate the forecasted net demand error.

\section{1)First Method: Forecast of the Day-ahead Net Demand Error}

The real ND is the difference of the sensed load and the sensed PV power. Based on the historical sensed and forecasted database of the load demand and PV production, the past forecasted ND is calculated as the difference between past forecasted load and past forecasted PV power at time step $h$. Then by using past real ND $\left(N D_{h-24}, \ldots, N D_{h-1}\right)$ and also past forecasted ND ( $\left.\tilde{N} D_{h-24}, \ldots, \tilde{N} D_{h-1}\right)$, the last $24 \mathrm{~h}$ prediction ND errors are obtained $\left(\varepsilon_{h-24}^{N D}, \ldots, \varepsilon_{h-1}^{N D}\right)$. Hence these data are used to calculate the day- 
ahead forecast of ND errors $\widetilde{\varepsilon}_{h}^{N D}(\mathrm{D}+1)$ (Fig. 3). The obtained ND error forecast can be characterized by the mean and variance (respectively, $\mu_{h}^{N D}$ and $\sigma_{h}^{N D}$ ).

\section{2)Second Method: Calculation from the PV Power and the Load Forecast Errors Estimation}

A second method is to define the ND uncertainty as the combination of PV power and load uncertainties. It is generally assumed that PV power and load forecast errors are unrelated random variables. So, firstly the day-ahead PV power and load forecasting errors $\left(\widetilde{\varepsilon}_{h}^{P V}\right.$ and $\widetilde{\varepsilon}_{h}^{L}$ ) are estimated independently. Then, last 24-hour load forecast errors and PV power forecast errors are calculated as the difference of the sensed load and PV power, and forecasted load and PV power, respectively (Fig. 4). The mean values and standard deviations of those forecasting errors can be obtained. Then the ND forecasting error can be attained as a new variable which comes from those two independent variables. The new obtained $p d f$ is also a normal distribution with the following mean and variance [20, 21]:

$\mu_{h}^{N D}=\mu_{h}^{L}-\mu_{h}^{P V}$

$\sigma_{h}^{N D}=\sqrt{\left(\sigma_{h}^{L}\right)^{2}+\left(\sigma_{h}^{P V}\right)^{2}}$

$\mu_{h}{ }^{L}$ and $\mu_{h}{ }^{P V}$ are respectively the mean values of load and PV power forecast errors prediction at time step $h, \sigma_{h}{ }^{L}$ and $\sigma_{h}{ }^{P V}$ are respectively the standard deviation square of the load and PV power forecasted errors prediction.

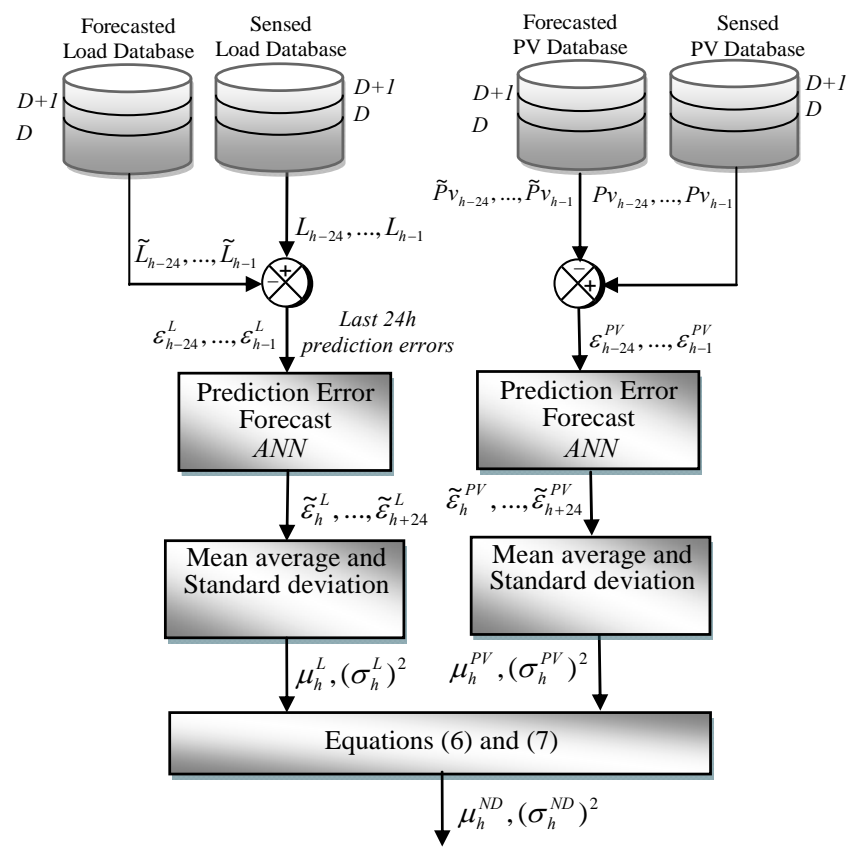

Fig. 4. Net demand uncertainty calculation from PV power and load forecasting errors prediction.

\section{B.Assessment of the Forecasting Uncertainty}

The predicted errors $\left(\widetilde{\varepsilon}_{h}^{N D}\right)$ of the ND forecast $\left(\tilde{N} D_{h}^{N D}\right)$ can be obtained with the normal probability density function (Fig. 5).

$p d f_{h}^{N D}=\frac{1}{\sigma_{h}^{N D} \sqrt{2 \pi}} \int_{-\infty}^{B} e^{\frac{-\left(\tau-\mu_{h}^{N D}\right)^{2}}{2\left(\sigma_{h}^{N D}\right)^{2}}} d \tau=F\left(B \mid \mu_{h}^{N D}, \sigma_{h}^{N D}\right)$

The forecasting uncertainty can be represented as upper and lower bound margins around the ND forecast. Bound margins $(B)$ are extracted by a normal inverse cumulative distribution function for a desired probability index $x$ (Fig. 5): 


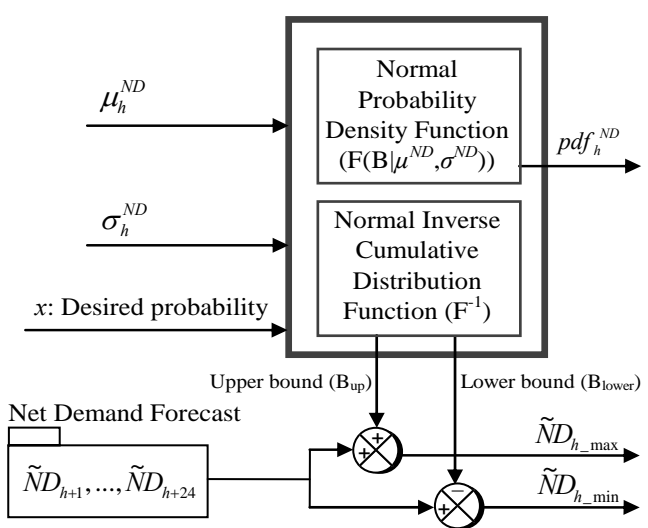

Fig. 5 Net uncertainty calculation at hour $h$ with a given probability.

\section{IV.POWER RESERVE QUANTIFICATION}

\section{A.Reliability Assessment}

Resulting from the uncertainty assessment, the $p d f$ of the forecasted ND errors in a given time step is considered for the calculation of the power reserve [23]. To estimate the impact of forecast ND uncertainty, two common reliability assessment parameters are used: the loss of load probability (LOLP) and the expected energy not served (EENS) [24-26]. LOLP represents the probability that the load demand $\left(L_{h}\right)$ exceeds PV power $\left(P_{h}\right)$ at time step $h$ :

$L O L P_{h}=\operatorname{prob}\left(L_{h}-P_{h}>0\right)=\int_{R}^{+\infty} p d f(\tau) \mathrm{d} \tau$

$\operatorname{prob}\left(L_{h}-P_{h}>0\right)$ is also the probability that the power reserve (R) is insufficient to satisfy the load demand in the time step $h$.

Meanwhile, EENS measures the magnitude of the load demand not served:

$\operatorname{EENS}_{h}=\operatorname{prob}\left(L_{h}-P_{h}>0\right) \times\left(L_{h}-P_{h}\right)$

where $\left(L_{h}-P_{h}\right)$ is the missed power in the time step $h$.

In this situation, the grid operator can either disconnect a part of loads or use the power reserve to increase the power production.

After obtaining each of the next 24 hours forecast ND $p d f_{s}$, an hourly day ahead reliability assessment can be attained. Electrical system operators can use this reliability to calculate the system security level.

\section{B.Risk-constrained Energy Management}

A reserve characteristic according to a risk level and for each time step can be obtained. With a fixed risk index, the operator can then easily quantify the power reserve [9]. As shown in Fig. 6, the sum of the shaded areas represents the accepted risk of violation with $x \%$ of LOLP. $R$ is the needed power reserve to compensate the remaining power unbalance. So, the reliability assessment can be done with the hourly cumulative distribution function ( $c d f$ ) obtained from the normal difference distribution of ND errors. Then the $c d f$ represents the probability that the random variable (here the ND error) is less or equal to $x$.

This assessment has been made under the assumption of a positive hourly forecasted ND. Otherwise, if the forecasted ND is negative, the reserve power for the same reliability level will be unnecessary (the power generation is more than the load demand). So the reliability has been assessed by considering only positive forecasted ND errors $\left(L_{h}-P_{h}\right)$ for each time step. Then, LOLP is deduced with: 
When the LOLP equals to the risk index $x \%$, the reserve power $(R)$ covers the remaining probability that the load demand exceeds the PV power generation (blue part in Fig. 6).

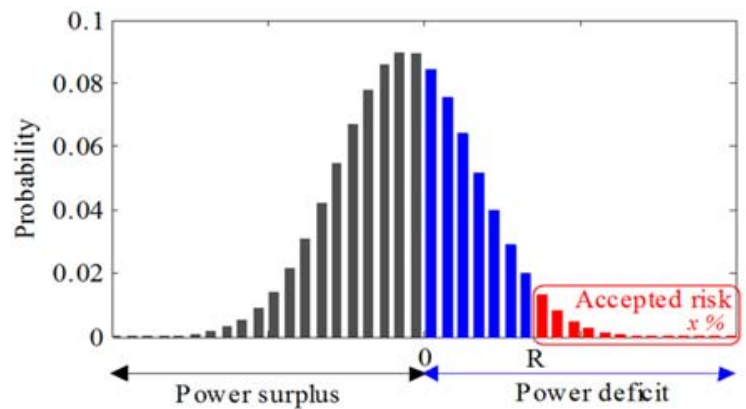

Fig. 6. Calculation of power reserve requirements $(R)$ based on forecast ND uncertainty $\left(\widetilde{\varepsilon}_{h}^{N D}\right)$ with $x \%$ of LOLP, at time step $h$.

\section{V.ILLUSTRATIVE CASE STUDY}

\section{A.Presentation and Data Collection}

The studied urban microgrid is a $110 \mathrm{~kW}$ load peak and is powered with $17 \mathrm{~kW}$ PV panels and three micro-gas turbines 30 $\mathrm{kW}, 30 \mathrm{~kW}$ and $60 \mathrm{~kW}$ each. Sensed data from our $17 \mathrm{~kW}$ PV plant located on the lab roof have been recorded in 2010 and 2013. For the load forecasting, past daily French power consumptions have been scaled to obtain per unit values of locally power consumption with the same characters and dynamics. A part of this database has been used to design the ANN based forecasting tool, a part to assess the estimation quality and a third one to implement the application of the proposed method in a real situation [15].

The ANN has been trained with past recorded data from the training set to predict hourly PV output power. The efficiency of the proposed method is validated by analyzing the normalized Root Mean Square Error (nRMSE) and normalized Mean Absolute Error $(n M A E)$ between predicted values $\left(\tilde{y}_{k}\right)$ and measured values $\left(y_{k}\right)$ :

$n R M S E=\sqrt{\frac{1}{n} \sum_{i=1}^{n}\left(\tilde{y}_{k}-y_{k}\right)^{2}}$

$n M A E=\frac{1}{n} \sum_{i=1}^{n}\left|\tilde{y}_{k}-y_{k}\right|$

\section{B.ANN Based Power Forecast and Net Demand Forecast}

\section{1)ANN based PV Power Forecasting}

A three-layer ANN has been developed for the PV power generation prediction with:

- one input layer including last $n$ hours of measured PV power, of irradiance and of forecasted average temperature (obtained from our local weather information service) (Fig. 7);

- one hidden layer with 170 neurons;

- one output layer with the 24 predicted PV power points (for each hour).

Various hidden layer neurons have been tested until getting an $n R M S E$ inferior to $5 \%$. First, $60 \%$ of previously sensed data (representing one year of data) have been used for training the ANN based PV power forecasting tool. Next $20 \%$ of sensed data are used to create a validation pattern set in order to assess the prediction quality. The test set (with the remaining 20\% data) is used to implement the forecast error calculation. Obtained $n R M S E$ and $n M A E$ for next 24 hours PV power predictions are given 
in Table I. Predicted errors for 120 test days are given in Fig. 8. Absolute values are less than 0.4 p.u. of the PV power output.
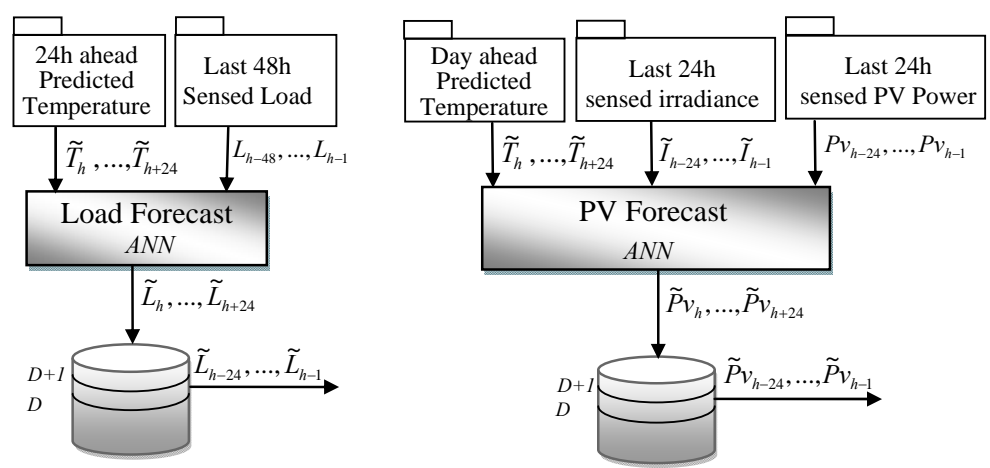

Fig. 7. PV power, load forecasting and errors prediction with ANN.

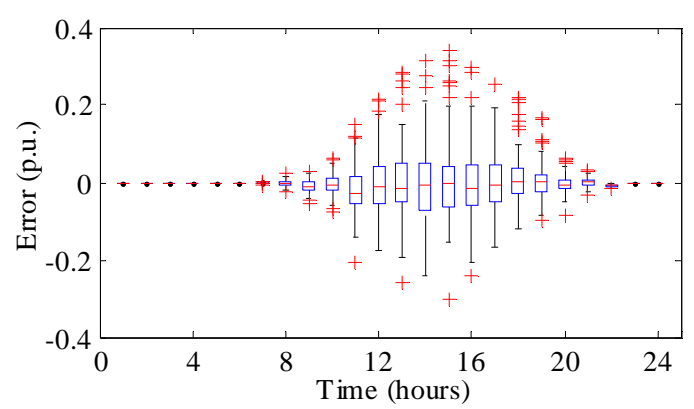

Fig. 8. PV power prediction errors on 120 test days.

TABLE I. Errors of the PV Power Forecast with ANN

\begin{tabular}{|c|c|c|}
\hline & $n R M S E[\%]$ & $n M A E[\%]$ \\
\hline Training Set & 4.67 & 2.69 \\
\hline Validation Set & 5.58 & 3.13 \\
\hline Test Set & 5.95 & 3.12 \\
\hline
\end{tabular}

\section{2)ANN based Load Forecasting}

Another neural network has been used for load forecast. The load demand prediction model includes: an input layer with last 48 hours load demand measurements and predicted temperatures for next 24 hours, one hidden layer with 70 neurons (in order to get an $n R M S E$ inferior to 4\%) and an output layer that predicts next 24 hours load demand. 60\% of available data are used for the neural network training, 20\% for the validation and 20\% for tests. The predicted errors for 120 test days are shown in Fig. 9. As it can be seen, the largest forecast error occurs at 8:00 and 18:00. Yet the total absolute errors are less than 0.2 p.u. of the load demand. Obtained results of nRMSE and nMAE are listed in Table II.

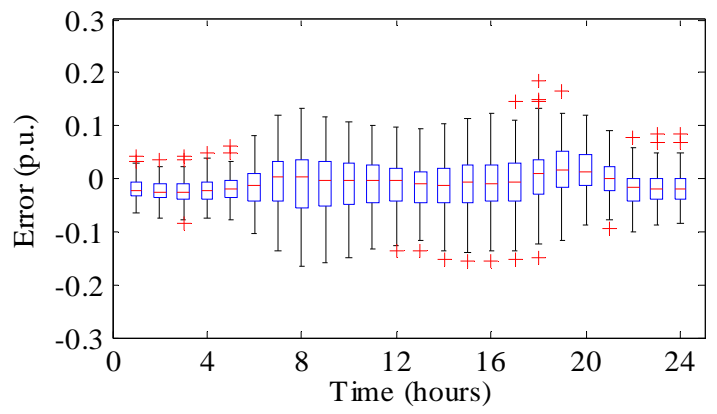

Fig. 9. Load prediction on 120 test days. 


\begin{tabular}{|c|c|c|}
\hline & $n R M S E[\%]$ & $n M A E[\%]$ \\
\hline Training Set & 3.18 & 2.45 \\
\hline Validation Set & 3.57 & 2.76 \\
\hline Test Set & 3.67 & 2.84 \\
\hline
\end{tabular}

\section{7}

3)Net Demand Uncertainty

\section{a) First Method: Direct Net Demand Forecast}

Following the method highlighted in Fig. 3, another ANN is applied for ND errors forecast: an input layer with last 24 hours predicted net demand errors, one hidden layer with 70 neurons and an output layer that predicts next 24 hours forecasted net errors.

Application of the first method (Fig. 3) for the time step at 12 am gives: $\mu_{12}^{N D}=-0.1282, \sigma_{12}^{N D}=1.781$ and the frequency distribution is shown on Fig 10(a).

\section{b) Second Method: Calculation of the PV Power and the Load Forecast Errors}

Two additional three-layer ANN are used to forecast the errors of PV power and load forecasts. Outputs are the predicted forecasting errors corresponding to the hourly predicted PV power and load, while inputs are the last 24 forecasting errors of PV power and loads.

For each hour, the mean and standard deviation have been calculated and the corresponding normal $p d f$ has been computed. As example, the distributions and normal $p d f$ of predicted errors of PV power and load errors forecast at 12 am are shown respectively in Fig. 10(b) with obtained parameters: $\mu_{12}^{P V}=-0.0353, \sigma_{12}^{P V}=0.01571$ for the PV forecast error $\left(\varepsilon_{h-12}^{P V}\right)$ and in Fig. 10(c) with obtained parameters: $\mu_{12}^{L}=-0.0353, \sigma_{12}^{L}=0.01571$ for the load forecast error $\left(\varepsilon_{h-12}^{L}\right)$.
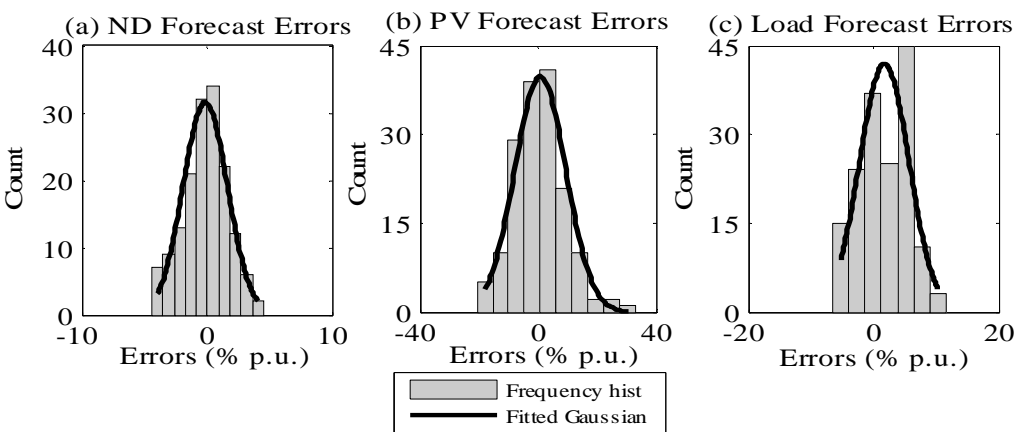

Fig. 10. Frequency distribution histograms and fitted Gaussian functions at $12 \mathrm{am}$.

\section{C.Forecasting Uncertainty Assessment}

By applying both proposed methods, the uncertainties of PV power forecasting, load forecasting and net forecasting with various probability indices (from $90 \%$ to $60 \%$ ) in a random day are represented as a function of the forecasting data and the predicted errors of forecasting. In order to simplify the explanation, results are given with the second method (corresponding to Fig. 5).

As shown in Fig. 11, the uncertainty of PV power forecasting is higher in the middle of the day, when the PV system generates the highest power. While in the morning (from 6:00 to 10:00) and afternoon (from 17:00 to 21:00), the uncertainty is smaller. Obviously, PV power forecasting uncertainty increases, and decreases with PV power increase and decrease respectively. Also, the uncertainty is increased when the time horizon is larger. For example, at 10:00 and at 17:00 power outputs are almost at the 
same level (about $6.5 \mathrm{~kW}$ ), but uncertainty is larger at 17:00 then at 9:00. The load forecasting has the same variation trend (Fig. 12).

Fig. 13 depicts the obtained ND uncertainty with the first method. If the forecasted ND is positive, then additional power sources have to be programmed to cover the difference. Otherwise, if forecasted ND is negative then three actions must be considered to meet the low forecasted demand:

- A part of PV power generators must be switched off (or can work at a sub-optimal level).

- Controllable loads (as electrical vehicles, heating loads.) must be switched on to absorb excess available power.

- Export the available excess energy to the main grid.

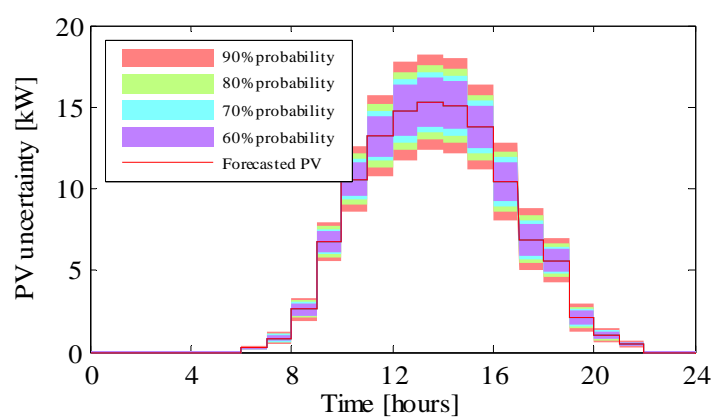

Fig. 11. PV forecasting with uncertainty (a random day).

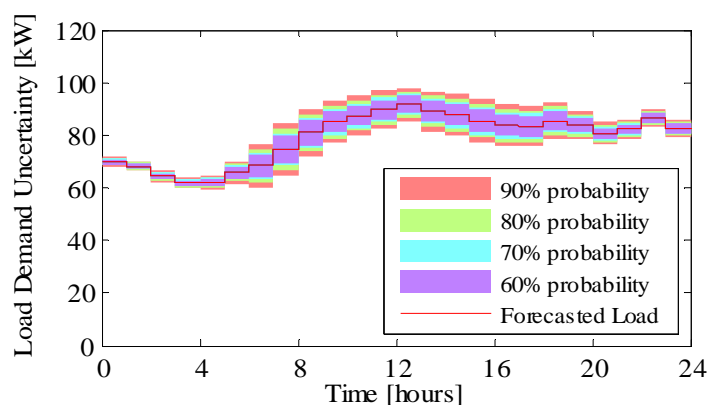

Fig. 12. Load forecasting with uncertainty (a random day).

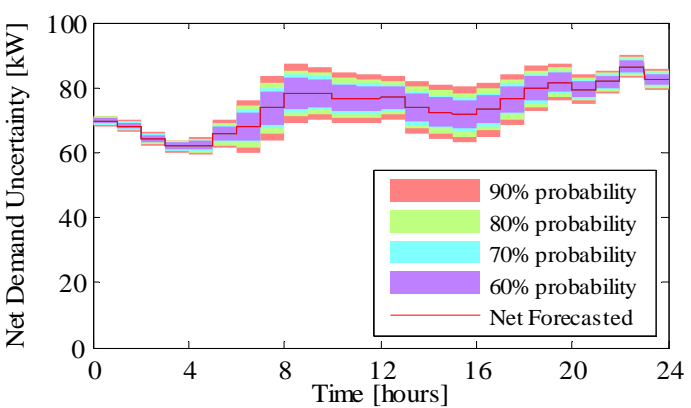

Fig. 13. Next 24 hours NFD with uncertainty (a random day).

\section{D.Power Reserve Calculation with Fixed Risk Indices}

The forecasted ND uncertainty assessment has been done with the hourly cumulative distribution function (cdf) obtained from the ND forecast errors. Then, the hourly risk/reserve curve takes into account all the errors from the $c d f_{s}$. Since the forecast ND errors can be expressed as an $x \%$ of the rated power, the PR can be drawn according to the LOLP. Fig. shows the required PR variation according to LOLP and EENS (with the second method in Section III). Therefore, an operating PR under $x$ \% of LOLP would cover a part of the forecast ND uncertainty. For example, with 10\% of LOLP, the reserve power will be $7 \mathrm{~kW}$ and the 
EENS will be $0.2 \mathrm{~kW}$. In general, this operating reserve is limited not only by the risk indices but also by the availability of microgas turbines.

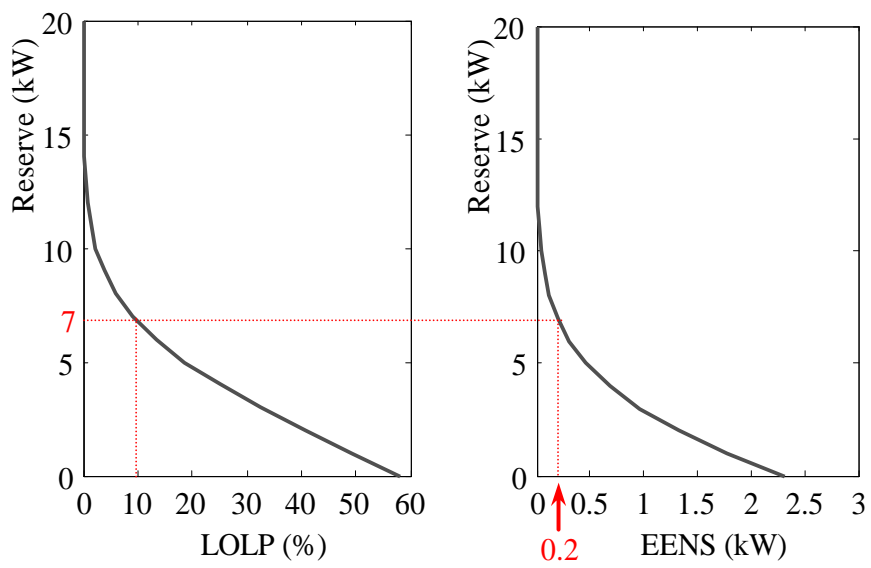

Fig. 14. Risk/reserve curve for $L O L P_{h+12}$ and $E E N S_{h+12}$ at 12:00.

On Fig. 15, an assessment of hourly reserve power required with the second method for different LOLP has been deduced. Much more reserve will be needed when the LOLP rate is very low, which means a high security level. While less reserve power will be needed with a high LOLP rate, but then the risk will be higher. For example with $1 \%$ of LOLP, the necessary PR will be $14 \mathrm{~kW}$ (EENS is almost zero) at 12:00 am, while the necessary reserve power will be $7 \mathrm{~kW}$ with a 10\% of LOLP and EENS increases to $0.25 \mathrm{kWh}$.

If a constant LOLP rate is set, the power reserve for each hour can be obtained. As shown in Fig. 16, with a 1\% of LOLP, more power reserve is needed in the middle of the day when larger PV power is generated. Moreover, power reserve with the second method is higher than the method with direct ND forecast. The most likely explanation of this result is because the load forecast uncertainty and PV forecast uncertainty are not totally independent. Sharing a common temperature, integrated PV power uncertainty and load uncertainty is greater than the direct ND forecast uncertainty. This result can be used for power dispatch management.

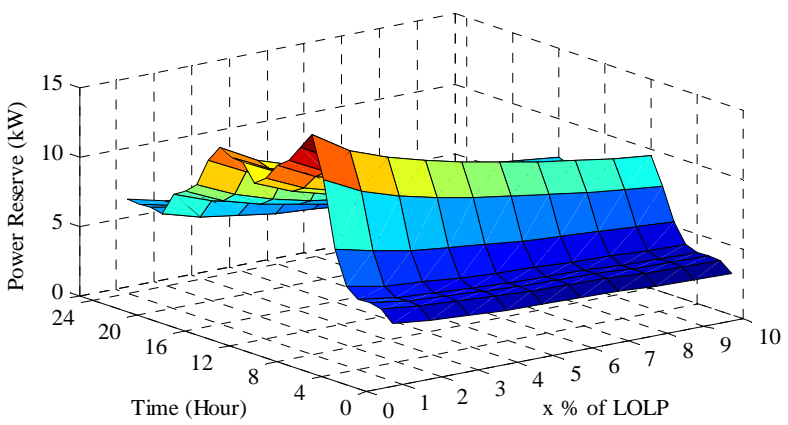

Fig. 15. Required power reserve for each hour with $x \%$ LOLP. 


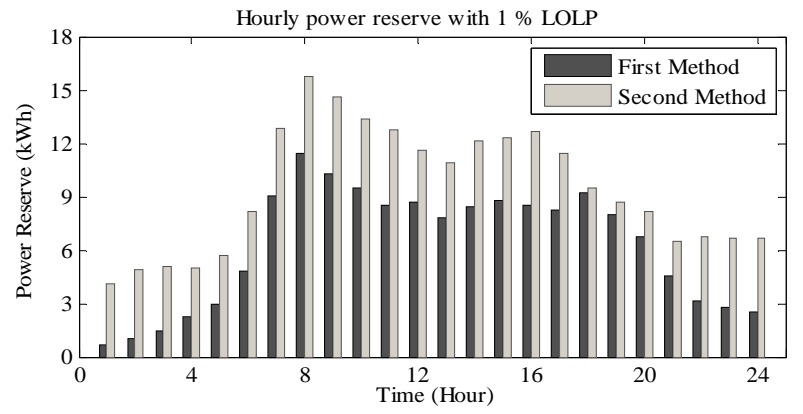

Fig. 16. Hourly power reserve with two different methods (1\% of LOLP).

\section{VI.CONCLUSION}

This work proposed a new technique to quantify the power reserve of a microgrid by taking into account the PV power forecasting uncertainty and load forecasting uncertainty. In order to assess these uncertainties, a three-layer BP ANN is used to estimate errors of PV power and load forecastings. Two methods are proposed to obtain the ND forecast uncertainties. With the first method, a probabilistic model is proposed to forecast ND uncertainty distribution by integrating the uncertainties from both PV power and load. The other method is desired to directly forecast the ND errors. The power reserve quantification results demonstrate that with a fixed risk index, the power reserve for next day or next 24 hours can be evaluated to cover the risk.

As the uncertainty from forecasting errors increases with time horizon, future research works are oriented toward the implementation of the intraday adjustment. The dispatch of the calculated power reserve onto micro-gas turbines, controllable loads and also new "PV based active power generators" is also an interesting way to pave.

\section{ACKNOWLEDGMENT}

The authors would like to thank the China Scholarship Council and Centrale Lille for their cofounding supports

\section{REFERENCES}

[1] E. Romero-Cadaval, B. Francois, M. Malinowski, and Q.-C. Zhong, "Grid-Connected Photovoltaic Plants: An Alternative Energy Source, Replacing Conventional Sources," Industrial Electronics Magazine, IEEE, vol. 9, pp. 18-32, 2015.

[2] A. Ipakchi and F. Albuyeh, "Grid of the future," Power and Energy Magazine, IEEE, vol. 7, pp. 52-62, 2009.

[3] F. D. Galiana, F. Bouffard, J. M. Arroyo, and J. F. Restrepo, "Scheduling and pricing of coupled energy and primary, secondary, and tertiary reserves," Proceedings of the IEEE, vol. 93, pp. 1970-1983, 2005.

[4] A. Mills, "Integrating Solar PV in Utility System Operations," Argonne National Laboratory, LBNL-6525E, Mar, 2014.

[5] A. Sobu and G. Wu, "Dynamic optimal schedule management method for microgrid system considering forecast errors of renewable power generations," in Power System Technology (POWERCON), 2012 IEEE International Conference on, 2012, pp. 1-6.

[6] M.-M. Buzau and B. Francois, "Quantification of operating power reserve through uncertainty analysis of a microgrid operating with wind generation," in Electrical Sciences and Technologies in Maghreb (CISTEM), 2014 International Conference on, 2014, pp. 1-8.

[7] X. Yan, B. Francois, and D. Abbes, "Operating power reserve quantification through PV generation uncertainty analysis of a microgrid," in PowerTech, 2015 IEEE Eindhoven, 2015, pp. 1-6.

[8] G. Delille, B. François, and G. Malarange, "Dynamic frequency control support by energy storage to reduce the impact of wind and solar generation on isolated power system's inertia," IEEE Transactions on Sustainable Energy vol. 3, pp. 931-939, 2012.

[9] K. De Vosa, A. G. Petoussis, J. Driesen, R. Belman, "Revision of reserve requirements following wind power integration in island power systems", Renewable Energy, vol. 50, Feb. 2013, pp. 268-279

[10] G. Sansavini, R. Piccinelli, L.R. Golea, E. Zio, "A stochastic framework for uncertainty analysis in electric power transmission systems with wind generation”, Renewable Energy, vol. 64, April 2014, pp. 71-81.

[11] H. Yang, J. Qiu, K. Meng, J. Hua Zhao, Z. Yang Dong, M. Lai, "Insurance strategy for mitigating power system operational risk introduced by wind power forecasting uncertainty", Renewable Energy, vol. 89, April 2016, Pages 606-615 
355

[12] M. Fuentes, G. Nofuentes, J. Aguilera, D. Talavera, and M. Castro, "Application and validation of algebraic methods to predict the behaviour of crystalline silicon PV modules in Mediterranean climates," Solar Energy, vol. 81, pp. 1396-1408, 2007.

[13] B. Espinar, J.-L. Aznarte, R. Girard, A. M. Moussa, and G. Kariniotakis, "Photovoltaic Forecasting: A state of the art," in Proceedings 5th European PVHybrid and Mini-Grid Conference, 2010.

[14] X. Yan, B. Francois, and D. Abbes, "Solar radiation forecasting using artificial neural network for local power reserve," in Electrical Sciences and Technologies in Maghreb (CISTEM), 2014 International Conference on, 2014, pp. 1-6.

[15] A. G. C. de Rocha Vaz, "Photovoltaic forecasting with artificial neural networks," University of Lisbon, 2014 (MSc thesis) Retrieved from, http://repositorio.ul.pt/bitstream/10451/11405/1/ulfc107351 tm_Andre_Vaz.pdf., 2014.

[16] A. A. Mohammed, W. Yaqub, and Z. Aung, "Probabilistic Forecasting of Solar Power: An Ensemble Learning Approach," in Intelligent Decision Technologies, ed: Springer, 2015, pp. 449-458.

[17] F. Golestaneh, H. B. Gooi, and P. Pinson, "Generation and evaluation of space-time trajectories of photovoltaic power," Applied Energy, vol. 176, pp. 8091, 2016.

[18] M. G. De Giorgi, P. M. Congedo, and M. Malvoni, "Photovoltaic power forecasting using statistical methods: impact of weather data," IET Science, Measurement \& Technology, vol. 8, pp. 90-97, 2014.

[19] L. Ghods and M. Kalantar, "Different methods of long-term electric load demand forecasting; a comprehensive review," Iranian Journal of Electrical \& Electronic Engineering, vol. 7, p. 249, 2011.

[20] M. A. Ortega-Vazquez and D. S. Kirschen, "Estimating the spinning reserve requirements in systems with significant wind power generation penetration," Power Systems, IEEE Transactions on, vol. 24, pp. 114-124, 2009.

[21] F. Bouffard and F. D. Galiana, "Stochastic security for operations planning with significant wind power generation," in Power and Energy Society General Meeting-Conversion and Delivery of Electrical Energy in the 21st Century, 2008 IEEE, 2008, pp. 1-11.

[22] B. Francois, "Orthogonal considerations in the design of neural networks for function approximation," Mathematics and computers in simulation, vol. 41, pp. 95-108, 1996.

[23] H. Holttinen, M. Milligan, E. Ela, N. Menemenlis, J. Dobschinski, B. Rawn, et al., "Methodologies to determine operating reserves due to increased wind power," in Power and Energy Society General Meeting (PES), 2013 IEEE, 2013, pp. 1-10.

[24] Y.-F. Li and E. Zio, "A multi-state model for the reliability assessment of a distributed generation system via universal generating function," Reliability Engineering \& System Safety, vol. 106, pp. 28-36, 2012.

[25] M. Wang and H. Gooi, "Spinning reserve estimation in microgrids," Power Systems, IEEE Transactions on, vol. 26, pp. 1164-1174, 2011.

[26] G. Liu and K. Tomsovic, "Quantifying spinning reserve in systems with significant wind power penetration," Power Systems, IEEE Transactions on, vol. 27, pp. 2385-2393, 2012.

[27] T. Hong, J. Wilson, and J. Xie, "Long term probabilistic load forecasting and normalization with hourly information," IEEE Transactions on Smart Grid, vol. 5, pp. 456-462, 2014. 